\title{
Immunolocalization of advanced glycation end products in human diabetic eyes: an immunohistochemical study
}

\author{
Satoru Kase ${ }^{1,3}$, Susumu Ishida ${ }^{3}$, Narsing Adupa Rao ${ }^{1,2}$ \\ ${ }^{1}$ Doheny Eye Institute, Los Angeles, USA; \\ ${ }^{2}$ Department of Ophthalmology, Keck School of Medicine of the University of Southern California, Los Angeles, USA; \\ ${ }^{3}$ Hokkaido University Graduate School of Medicine, Sapporo, Japan; ${ }^{*}$ Corresponding Author: kaseron@med.hokudai.ac.jp
}

Received 25 May 2011; revised 6 July 2011; accepted 14 July 2011.

\begin{abstract}
Background: Advanced glycation end-products (AGEs) play a critical role in the pathology of diabetic complications. The aim of this study is to examine the immunolocalization of advanced glycation end products (AGE) and receptor for AGE (RAGE) in human diabetic and non-diabetic donor eyes using immunohistochemistry. Materials and Methods: Eight globes were obtained from human postmortem donors: six diabetic donors and two non-diabetic. Formalin-fixed, paraffin-embedded tissue sections were subjected to immunohistochemistry with anti-AGE, and RAGE antibodies. Results: In eyes from diabetic donors, the blood vessels of the iris and choroid had relatively thickened walls. The ciliary body showed decreased capillaries with hyalinization in the stroma. Neovascularization or proliferative changes were not observed in the tissues. Immunoreactivity for AGE was highly detected in the stroma and blood vessels of the iris, ciliary body, choriocapillaris, choroidal large vessels, and central retinal artery/vein. Immunoreactivity was also detected in the retina, corneal endothelium, and lens. RAGE immunoreactivity was weakly detected in choroidal vessels and Bruch's membrane. In eyes from non-diabetic donors, AGE was weakly detected in the iris, ciliary body stroma, and choriocapillaris, but RAGE was hardly detected. Conclusion: AGE is highly accumulated in vascularized intraocular tissues of diabetic eyes, suggesting that AGE accumulation may play an important role in the pathogenesis of diabetic vasculopathy. This study indicates that inhibition of AGE formation may be an important therapeutic strategy for sup-
\end{abstract}

pressing the progression of diabetic ocular complications.

Keywords: Advanced Glycation End Products; Choroid; Ciliary Body; Diabetes; Iris; Retina

\section{INTRODUCTION}

Diabetic vascular complications are a major cause of acquired blindness in type 1 and type 2 diabetes mellitus [1]. Studies have confirmed that hyperglycemia, leading to metabolic and hemodynamic derangement, is the most important factor in the onset and progress of vascular complications in diabetes mellitus [2,3]. Diabetic microangiopathy is characterized by blood retinal barrier breakdown, capillary basement membrane thickening, loss of pericytes, and development of acellular and occluded capillaries [4,5]. On the other hand, the pathological alterations associated with diabetes mellitus are found in various intraocular tissues [6], including the vascularized tissues of the iris, ciliary body, choroid, and retina, as well as the non-vascularized tissues of the cornea and lens. These suggest that diabetic ocular complications are caused, either directly or indirectly, by microangiopathy.

The formation of advanced glycation end-products (AGEs) correlates with glycemic control [7]. AGE may cause tissue injury both directly, through phenomena such as trapping and cross-linking, and indirectly, by binding to the specific receptor for AGE (RAGE) on the surface of various cells [8]. The pathological crosslink formation induced by AGE usually affects the stable and long-lived proteins, leading to increased stiffness of the protein matrix and increased resistance to removal by proteolytic means [8-10]. In subsequent investigations, a high concentration of AGE was noted in the vitreous and aqueous humor in patients with diabetic retinopathy [11,12]. The increase in AGE colocalized with RAGE in 
the diabetic retina is correlated with the onset and progression of retinopathy [13]. AGE accumulation has been evaluated by immunohistochemistry in the cornea, retina, optic nerve head, and Bruch's membrane of human diabetic donor eyes [14-16]. Moreover, corneal AGE fluorescence values, measured by fluorophotometry, were markedly higher in the patients with proliferative diabetic retinopathy than in the control subjects [17]. However, immunolocalization of AGE has yet to be elucidated enough in human vascularized intraocular tissues.

The present study examined the immunolocalization of AGE and RAGE in human diabetic and non-diabetic donor eyes. The immunoreactivity was then compared in vascularized and non-vascularized ocular tissues.

\section{MATERIALS AND METHODS}

\subsection{Preparation of Samples}

The institutional review board of the University of Southern California approved our use of human specimens obtained from the files of the Doheny Eye Institute Pathology Laboratory. All procedures conformed to the Declaration of Helsinki for research involving human subjects. Eight eyes were obtained from the Doheny Eye and Tissue Transplant Bank, Los Angeles, CA. These eight eyes were from four donors who died as a result of cardiac-related disorders. Six of the eight eyes came from three donors with a history of diabetes mellitus; the remaining two eyes came from a donor with no history of diabetes. The clinical data examined in this study are presented in Table 1. All eight eyeballs were fixed in 4\% paraformaldehyde soon after enucleation.

\subsection{Immunohistochemistry}

The slides were dewaxed, rehydrated, and rinsed in phosphate-buffered saline twice for $10 \mathrm{~min}$. As pretreatment, microwave-based antigen retrieval was performed in $10 \mathrm{mM}$ citrate buffer ( $\mathrm{pH}$ 6.0). These slides were incubated with $3 \%$ hydrogen peroxide for $10 \mathrm{~min}$, then with normal goat serum for $30 \mathrm{~min}$. Sections were incubated with anti-AGE (1:100; ab23722; Abcam, Cambridge, MA), and RAGE (1:100; ab37647; Abcam) rabbit polyclonal antibodies at room temperature for $2 \mathrm{hr}$. Binding of the primary antibody was localized with the FITC-conjugated anti-rabbit secondary antibody (Jackson ImmunoResearch Laboratories, West Grove, PA) for $30 \mathrm{~min}$. Negative control consisted of FITC-conjugated mouse IgG incubated without treatment of the primary antibody. Slides were examined using a Zeiss LSM510 (Zeiss, Thornwood, NY) confocal microscope.

\section{RESULTS}

\subsection{AGE is Highly Accumulated in the Iris Stroma in Diabetic Eyes}

The iris consisted of one layer of pigmented epithelium and the stroma. In diabetic donor globes, the iris stromal vessels contained slightly thickened walls (Figure 1(a)). Pigmentation was noted in the stroma, whereas neovascularization was not observed in diabetic iris. Periodic acid Schiff (PAS) staining was weakly positive for vascular walls in diabetic eyes. In contrast, several blood vessels with thin vessel walls and clear vascular lumens were distributed in the stroma of non-diabetic eye (Figure 1(e)). Immunoreactivity for AGE was strongly detected in the iris stroma, including the blood vessels in the diabetic donor eyes (Figures 1(b) and (c)), whereas the immunoreactivity was less marked in non-diabetic donor eyes (Figures 1(f) and (g)). Although linear membranous immunoreactivity for AGE was weakly observed, cytoplasmic immunoreactivity was not seen in pigmented epithelium of diabetic (Figure 1(b)) and non-diabetic donor eyes (Figure 1(f)). AGE immunoreactivity was weakly detected in the sphincter muscle of the iris. Immunoreactivity for RAGE was not detected in diabetic (Figure 1(d)) or non-diabetic iris (Figure 1(h)).

\subsection{AGE Is Highly Accumulated in the Ciliary Body Stroma of Diabetic Eyes}

The ciliary body consisted of two layers of epithelium and the stroma. In the diabetic globes, the basement membrane of the ciliary body stroma was thickened (Figure 2(a)). The stroma displayed hyalinization, where the number of core capillaries was decreased (Figure 2(a), arrows). PAS staining clearly revealed thickening of the pigmented ciliary body epithelial basement membrane of the pars plicata (Figure 2(b)). In contrast, blood vessels showing clear vascular lumens were distributed in the stroma of non-diabetic eyes (Figure 2(e), arrows). PAS staining was weakly positive for basement membrane of epithelium in non-diabetic globes (Figure 2(f)). Immunoreactivity for AGE was strongly detected in the ciliary body stroma, including the blood vessels, in the diabetic eyes (Figure 2(c)), whereas the immunoreactivity was less marked in non-diabetic eyes (Figure 2(g)). Although weak immunoreactivity for AGE was detected in the cell membrane of the apical side, no cytoplasmic immunoreactivity was seen in the ciliary body epithelia of diabetic or non-diabetic eyes. AGE immunoreactivity was not seen in blood vessels situated in the stroma of non-diabetic eyes (Figure 2(g); arrows). No immunoreactivity for RAGE was detected in either the diabetic (Figure 2(d)) or non-diabetic ciliary body (Figure 2(h)). 
Table 1. Clinicopathological profile in human donor eyes examined in this study.

\begin{tabular}{|c|c|c|c|c|c|c|c|c|c|c|c|c|c|c|}
\hline No. & Age & Sex & DM & Type & Duration & Iris & $\begin{array}{c}\text { Ciliary } \\
\text { body }\end{array}$ & Choroid & Retina & Cornea & Cornea & Lens & Sclera & Optic nerve \\
\hline & & & & & (years) & & & & & epithelium & ndotheliu & & & \\
\hline 1 & 53 & M & + & IDDM & 9 & +++ & +++ & +++ & ++ & - & +++ & + & +++ & +++ \\
\hline 2 & 69 & $\mathrm{~F}$ & + & IDDM & 10 & +++ & ++ & +++ & + & - & ++ & + & +++ & + \\
\hline 3 & 39 & M & + & NIDDM & 2 & +++ & +++ & +++ & + & - & + & + & + & ++ \\
\hline 4 & 70 & F & - & - & 0 & + & + & + & + & - & + & + & + & - \\
\hline
\end{tabular}

M, male; F, female; DM, diabetes mellitus; IDDM, insulin-dependent diabetes mellitus; NIDDM, non-IDDM; AGE, advanced glycation end product; +, weak; ++ , moderate; +++ , strong positive.

\subsection{AGE Is Highly Accumulated in the Choroid in Diabetic Eyes}

Histopathologic examination revealed thickening of the large choroidal vessel walls in diabetic eyes (Figure 3(a)), whereas the vessel wall thickening was not observed in non-diabetic eyes (Figure 3(e)). AGE immunoreactivity was strongly detected in the choriocapillaris and large choroidal vessels, as well as in the stroma (Figures 3(b) and (c)). In non-diabetic eyes, AGE expression was weakly detected in the choriocapillaris, but the expression in large vessels and the stroma was less marked (Figures 3(f) and (g)). Immunoreactivity for RAGE was detected in the Bruch's membrane and large choroidal vessels in diabetic eyes (Figure 3(d)), whereas the immunoreactivity was less marked in non-diabetic eyes (Figure $\mathbf{3 ( h )}$ ).

\subsection{Expression of AGE in Central Retinal Artery/Vein and the Retina}

AGE was highly accumulated in the vessel walls of the central retinal artery and vein, located upon the optic nerve head, of diabetic eyes (Figures 4(a) and (b)), whereas the immunoreaction was less marked in non-diabetic eyes (Figures 4(d) and (e)). AGE immunoreactivity was weakly detected in the retina, including the inner limiting membrane and the inner and outer nuclear layers, in diabetic (Figures 4(g) and (h)) and non-diabetic eyes (Figures 4(i) and (k)). AGE immunoreactivity was also observed in the vessel walls of the peripheral retina in both groups (Figures 4(g)-(j): arrow) and in the vitreous of diabetic eyes (Figures 4(g) and (h)): asterisk), but was not seen in the vitreous of non-diabetic eyes (Figures 4(j) and (k)). RAGE was not expressed in the central retinal artery/vein or the retina in diabetic (Figures 4(c) and (i)) or non-diabetic (Figures 4(f) and (l)) eyes.

\subsection{Comparison of AGE Immunoreactivity with Other Ocular Tissues}

In diabetic eyes, AGE was expressed in corneal endothelium, pia mater, and parenchyma of the optic nerve, and sclera. Lens epithelial cells showed faint expression of AGE (data not shown). As shown in Table 1, AGE immunoreactivity was strongly detected in the iris, ciliary body, choroid, and central retinal vessels, while the immunoreactivity was relatively weak in the corneal endothelium, sclera, and retina in diabetic eyes (Table 1). Expression of RAGE was not detected in diabetic ocular tissues, except Bruch's membrane and large choroidal vessels

\section{DISCUSSION}

Histopathologic examination revealed thickened basement membrane, decreased number of endothelial cells,

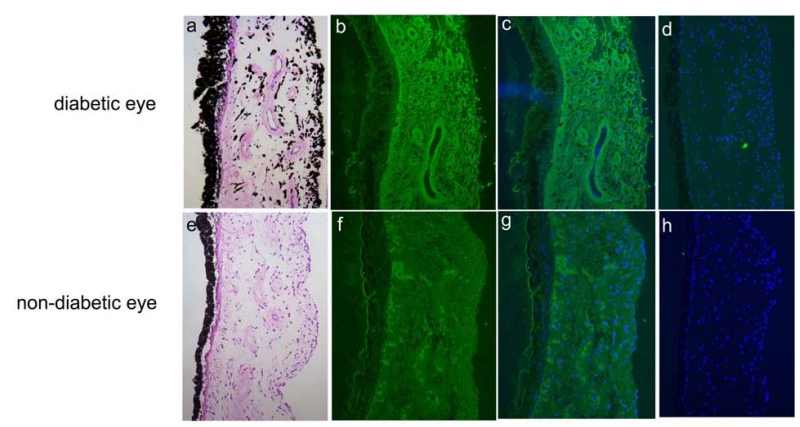

Figure 1. Hematoxylin \& eosin (HE) (a, e) and immunodetection of advanced glycation end product (AGE) (b, c, f, g; green) and receptor for AGE (RAGE) (d, h; green) with DAPI nuclear staining (c, d, g, h; blue) in the iris in human diabetic (a-d) and non-diabetic (e-h) eyes.

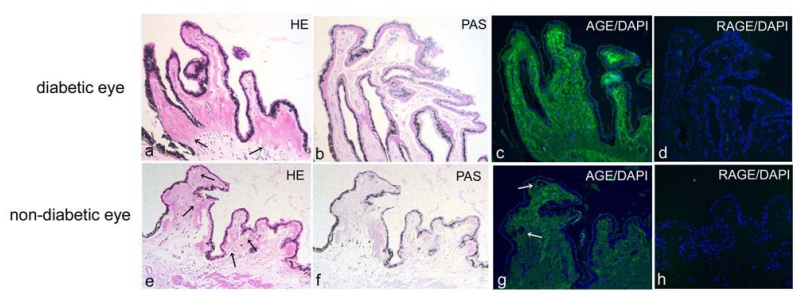

Figure 2. Hematoxylin \& eosin (HE) (a, e) and periodic acid Schiff (PAS) staining (b, f), and immunodetection of advanced glycation end product (AGE) (c, g; green) and receptor for AGE (RAGE) (d, h; green) with DAPI nuclear staining (c, d, g, $\mathrm{h}$; blue) in the pars plicata of ciliary body in human diabetic (a-d) and non-diabetic (e-h) eyes. 


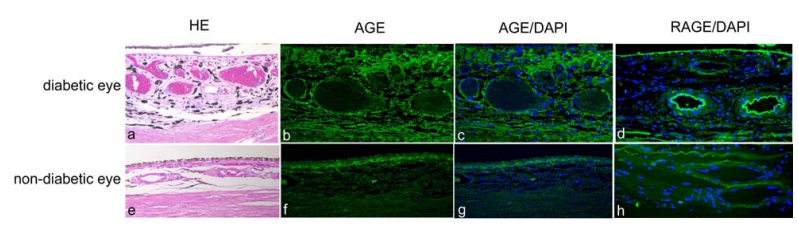

Figure 3. Hematoxylin \& eosin (HE) (a, e) and immunodetection of advanced glycation end product (AGE) (b, c, f, g; green) and receptor for AGE (RAGE) (d, h; green) with DAPI nuclear staining (c, d, g, h; blue) in the choroid in human diabetic (a-d) and non-diabetic (e-h) eyes.

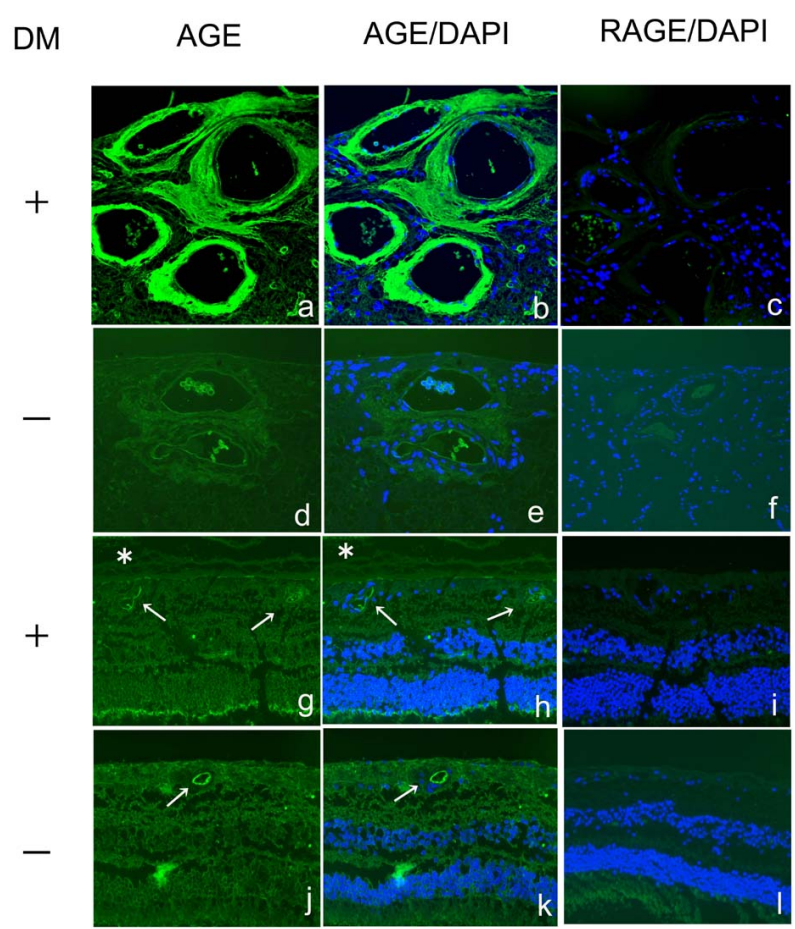

Figure 4. Immunodetection of advanced glycation end product (AGE) (a, b, d, e, g, h, j, k; green) and receptor for AGE (RAGE) (c, f, i, l; green) with DAPI nuclear staining (b, c, e, f, $h, i, k, l$; blue) in the retina and the vessels in human diabetic (a-c, g-i) and non-diabetic (d-f, j-l) eyes. DM, diabetes mellitus.

and increased acellular areas in the ciliary body stroma of human diabetic globe; these findings were not observed in the non-diabetic globes. The blood vessels of the iris stroma in the diabetic eyes had relatively thickened walls compared to those in non-diabetic eyes. These pathological findings indicate that alteration of blood vessels in the iris and ciliary body clearly reflects diabetic microangiopathy [4,5]. Immunoreactivity for AGE was strongly detected in the stromal vessels of the iris and ciliary body, as well as in the choriocapillaris and peripheral retinal vessels, of diabetic eyes, whereas AGE expression was less marked in the non-diabetic eyes. Moreover, AGE expression was stronger in the ciliary body, iris, and choriocapillaris than in the other ocular tissues, including the cornea and lens. Therefore, AGE is predominantly accumulated in the preexisting well-vascularized ocular tissues of diabetes, implying that AGE is primarily accumulated during the pathogenesis of diabetic microangiopathy.

High concentrations of AGE have been observed in the vitreous of patients with diabetic retinopathy $[11,12,18]$. This immunohistochemical study showed that AGE immunoreactivity was detectable in the vitreous of diabetic eyes, suggesting that AGE can be secreted into intraocular fluid. In addition, Endo et al. demonstrated that AGE concentration was increased in the aqueous humor in diabetic patients without retinopathy [11]. This study clearly demonstrated that AGE accumulation was marked in the iris and ciliary body stroma of diabetic eyes, indicating that the high concentration of AGE in the aqueous humor is related to the accumulated AGE in the iris and ciliary body. We also confirmed that AGE was expressed in the corneal endothelium of two diabetic eyes compared to age-matched non-diabetic eyes, as shown in Table 1. Although Kaji et al. showed AGE expression in corneal endothelium, they speculated that AGE accumulation might result from the decrease in corneal endothelial cells that occurs with aging [14]. The current study suggests that high expression and concentration of AGE in the iris and the aqueous humor may directly involve corneal endothelium, subsequently leading to increased AGE expression.

AGE is accumulated at many sites of the body, including the heart and large blood vessels, in diabetes [19]. Interestingly, we also demonstrated that AGE accumulation was noted not only in microvessels located in the diabetic retina and uveal tract, but also in relatively large vessels, including the central retinal artery/vein and the choroid. Occlusion of the central retinal artery/vein is a known ocular complication of diabetes [20]. Hyperglycemia leads to matrix metalloproteinase expression via AGE formation, which subsequently contributes to histological alterations and accelerated plaque formation in large vessels [19,21]. These findings suggest that AGE plays a potential role in the pathogenesis, not only of microangiopathy, but of retinal vessel occlusion in diabetes.

We found that AGE accumulated in the blood vessels, as well as in the stroma of the iris and ciliary body. Diabetes mellitus can complicate uveitis representing diabetic iridocyclitis [22]; however, the pathogenesis remains unknown. It is known that hyperglycemia promotes inflammation through the induction of cytokine secretion by several cell types including monocytes. AGE induces the expression of pro-inflammatory molecules through mitogen-activated protein kinase and nu- 
clear factor kappaB-dependent pathways [23]. Furthermore, AGE is believed to play an important role in the onset of uveitis in Vogt-Koyanagi-Harada disease [24]. Our results support the hypothesis that a strong accumulation of AGE in the iris and ciliary body in diabetic eyes may have a causative effect on the onset of diabetic iridocyclitis.

RAGE expression has been detected in the glial cells and vascular endothelial cells intermingled in the epiretinal membrane of proliferative diabetic retinopathy [25]. In the present study, RAGE expression was detected in Bruch's membrane and large choroidal vessels; but no RAGE expression was noted in the retinal vessels of either diabetic or non-diabetic eyes. This suggests that RAGE may be strongly expressed during the development of diabetic retinopathy. AGE may cause tissue injury both directly and indirectly by binding to RAGE on the surface of various cells [8]. The present no RAGE expression was observed in the uveal tracts or central retinal vessels, places where AGE was highly accumulated. These suggest that the pathogenesis of diabetic vasculopathy is due to direct tissue injury through accumulated AGE rather than binding to the receptor.

AGE is now believed to contribute to the accelerated microvasculopathy observed in diabetes [1]. We clearly demonstrated that AGE was accumulated in diabetic well-vascularized ocular tissues. Inhibition of AGE pathway, including AGE formation, AGE-RAGE interaction, and RAGE expression, has been considered a therapeutic molecular targeting. Indeed, agents that inhibit the formation of AGE have been shown to clinically suppress the progression in diabetic vascular complications [1]. The current immunohistochemical analysis suggests that inhibition of AGE formation is an important therapeutic strategy to suppress the onset of diabetic retinopathy.

\section{ACKNOWLEDGEMENTS}

Supported by NIH grants EY017347, EY015714 and EY03040 and by an unrestricted grant from Research to Prevent Blindness.

\section{REFERENCES}

[1] Yamagishi, S., Nakamura, K., Matsui, T., Ueda, S., Fukami, K. and Okuda, S. (2008) Agents that block advanced glycation end product (AGE)-RAGE (receptor for AGEs)-oxidative stress system: A novel therapeutic strategy for diabetic vascular complications. Expert Opinion on Investigational Drugs, 17, 983-996. doi:10.1517/13543784.17.7.983

[2] Brownlee, M. (2001) Biochemistry and molecular cell biology of diabetic complications. Nature, 414, 813-820. doi:10.1038/414813a

[3] Yamagishi, S. and Imaizumi, T. (2005) Diabetic vascular complications: Pathophysiology, biochemical basis and potential therapeutic strategy. Current Pharmaceutical Design, 11, 2279-2299. doi:10.2174/1381612054367300

[4] De La Cruz, J.P., Gonzalez-Correa, J.A., Guerrero, A. and de la Cuesta, F.S. (2004) Pharmacological approach to diabetic retinopathy. Diabetes/Metabolism Research and Reviews, 20, 91-113. doi:10.1002/dmrr.432

[5] Donnelly, R., Emslie-Smith A.M., Gardner I.D. and Morris, A.D. (2000) ABC of arterial and venous disease: Vascular complications of diabetes. British Medical Journal, 320, 1062-1066.

doi:10.1136/bmj.320.7241.1062

[6] Yanoff, M. and Fine, B. (2002) Ocular pathology, Fifth Ediyion, Mosby, St. Louis.

[7] Fu, M.X., Requena, J.R., Jenkins, A,J,, Lyons, T.J., Baynes, J.W. and Thorpe, S.R. (1996) The advanced glycation end product, Nepsilon-(carboxymethyl)lysine, is a product of both lipid peroxidation and glycoxidation reactions. The Journal of Biological Chemistry, 271, 9982-9986. doi:10.1074/jbc.271.17.9982

[8] Diamanti-Kandarakis, E., Piperi, C., Patsouris, E., Korkolopoulou, P., Panidis, D., Pawelczyk, L., Papavassiliou, A.G. and Duleba, A.J. (2007) Immunohistochemical localization of advanced glycation end-products (AGEs) and their receptor (RAGE) in polycystic and normal ovaries. Histochemistry and Cell Biology, 127, 581-589. doi:10.1007/s00418-006-0265-3

[9] Thomas, M.C., Forbes, J.M. and Cooper, M.E. (2005) Advanced glycation end products and diabetic nephropathy. American Journal of Therapeutics, 12, 562-572. doi:10.1097/01.mjt.0000178769.52610.69

[10] Calabrese, V., Mancuso, C., Sapienza, M., Puleo, E., Calafato, S., Cornelius, C., Finocchiaro, M., Mangiameli, A., Di Mauro, M., Stella, A.M. and Castellino, P. (2007) Oxidative stress and cellular stress response in diabetic nephropathy. Cell Stress and Chaperones, 12, 299-306. doi:10.1379/CSC-270.1

[11] Endo, M., Yanagisawa, K., Tsuchida, K., Okamoto, T., Matsushita, T., Higuchi, M., Matsuda, A., Takeuchi, M., Makita, Z. and Koike, T. (2001) Increased levels of vascular endothelial growth factor and advanced glycation end products in aqueous humor of patients with diabetic retinopathy. Hormone Metabolism Research, 33, 317-322. doi:10.1055/s-2001-15122

[12] Stitt, A.W., Moore, J.E., Sharkey, J.A., Murphy, G., Simpson, D.A., Bucala, R., Vlassara, H. and Archer D.B. (1998) Advanced glycation end products in vitreous: Structural and functional implications for diabetic vitreopathy. Investigative Ophthalmology \& Visual Science, 39, 2517-2523.

[13] Stitt, A.W., Li, Y.M., Gardiner, T.A., Bucala, R., Archer, D.B. and Vlassara, H. (1997) Advanced glycation end products (AGEs) co-localize with AGE receptors in the retinal vasculature of diabetic and of AGE-infused rats. American Society for Investigative Pathology, 150, 523531.

[14] Kaji, Y., Usui, T., Oshika, T., Matsubara, M., Yamashita, H., Araie, M., Murata, T., Ishibashi, T., Nagai, R., Horiuchi, S. and Amano, S. (2000) Advanced glycation end products in diabetic corneas. Investigative Ophthalmology \& Visual Science, 41, 362-368.

[15] Amano, S., Kaji, Y., Oshika, T., Oka, T., Machinami, R., Nagai, R. and Horiuchi, S. (2001) Advanced glycation 
end products in human optic nerve head. British Journal of Ophthalmology, 85, 52-55. doi:10.1136/bjo.85.1.52

[16] Handa, J.T., Verzijl, N., Matsunaga, H., Aotaki-Keen, A., Lutty, G.A., te Koppele, J.M., Miyata, T. and Hjelmeland, L.M. (1999) Increase in the advanced glycation end product pentosidine in Bruch's membrane with age. Investigative Ophthalmology \& Visual Science, 40, 775-779.

[17] Sato, E., Mori, F., Igarashi, S., Abiko, T., Takeda, M., Ishiko, S. and Yoshida, A. (2001) Corneal advanced glycation end products increase in patients with proliferative diabetic retinopathy. Diabetes Care, 24, 479-482. doi:10.2337/diacare.24.3.479

[18] Yokoi, M., Yamagishi, S.I., Takeuchi, M., Ohgami, K., Okamoto, T., Saito, W., Muramatsu, M., Imaizumi, T. and Ohno, S. (2005) Elevations of AGE and vascular endothelial growth factor with decreased total antioxidant status in the vitreous fluid of diabetic patients with retinopathy. British Journal of Ophthalmology, 89, 673-675. doi:10.1136/bjo.2004.055053

[19] Jandeleit-Dahm, K. and Cooper, M.E. (2008) The role of AGEs in cardiovascular disease. Current Pharmaceutical Design, 14, 979-986. doi:10.2174/138161208784139684

[20] Watkinson, S. and Seewoodhary, R. (2008) Ocular complications associated with diabetes mellitus. Nursing Standard, 22, 51-57.

[21] Kadoglou, N.P., Daskalopoulou, S.S., Perrea, D. and Liapis, C.D. (2005) Matrix metalloproteinases and dia- betic vascular complications. Angiology, 56, 173-189. doi:10.1177/000331970505600208

[22] Goto, H., Mochizuki, M., Yamaki, K., Kotake, S., Usui, M. and Ohno, S. (2007) Epidemiological survey of intraocular inflammation in Japan. Japanese Journal of Ophthalmology, 51, 41-44. doi:10.1007/s10384-006-0383-4

[23] Lappas, M., Permezel, M. and Rice, G.E. (2007) Advanced glycation endproducts mediate pro-inflammatory actions in human gestational tissues via nuclear factor-kappaB and extracellular signal-regulated kinase 1/2. Journal of Endocrinology, 193, 269-277. doi:10.1677/JOE-06-0081

[24] Kitamura, M., Kitaichi, N., Takeuchi, M., Kitamei, H., Namba, K., Yamagishi, S.I., Iwabuchi, K., Onoé and K. Ohno, S. (2005) Decrease in the glyceraldehyde derived advanced glycation end products in the sera of patients with Vogt-Koyanagi-Harada disease. British Journal of Ophthalmology, 89, 1407-1409. doi:10.1136/bjo.2005.072678

[25] Pachydaki, S.I., Tari, S.R., Lee, S.E., Ma, W., Tseng, J.J., Sosunov, A.A., Cataldergirmen, G., Scarmeas, N., Caspersen, C., Chang, S., Schiff, W.M., Schmidt, A.M. and Barile, G.R. (2006) Upregulation of RAGE and its ligands in proliferative retinal disease. Experimental Eye Research, 82, 807-815. doi:10.1016/j.exer.2005.09.022 